Audiology

Neurotology
Audiol Neurotol 2016;21:244-249

DOI: $10.1159 / 000445736$
Received: December 3, 2015

Accepted after revision: March 22, 2016

Published online: August 30, 2016

\title{
Force Perception Thresholds in Cochlear Implantation Surgery
}

\author{
Louis B. Kratchman Daniel Schuster Mary S. Dietrich Robert F. Labadie \\ Vanderbilt University and Vanderbilt University Medical Center, Nashville, Tenn., USA
}

\section{Key Words}

Cochlear implantation surgery · Trauma - Insertion forces ·

Psychophysics $\cdot$ Tactile perception

\begin{abstract}
Tissue trauma is a frequent complication of cochlear implantation $(\mathrm{Cl})$ surgery, but the relationship between intracochlear trauma, electrode insertion forces, and surgeons' ability to perceive these forces is poorly understood. In this study, we simulated $\mathrm{Cl}$ surgery using a benchtop apparatus to repeatedly apply small forces to the subjects' hands while reducing the variability in their hand movements. We used a psychophysical testing procedure to estimate the force perception thresholds of 10 otologic surgeons and found a median threshold of $20.4 \mathrm{mN}$. The results suggest that surgeons have the capability to sense at least some insertion forces and are likely to perceive severe trauma such as occurs when the electrode crosses from one scala to the other.
\end{abstract}

(C) 2016 S. Karger AG, Basel

\section{Introduction}

Cochlear implants have been described as the most successful neural prostheses [Wilson and Dorman, 2008], yet estimates of complication rates in the literature range from 4 to $40 \%$ [Brito et al., 2012], and suboptimal hearing outcomes are one potential effect of these complications. Surgeons do not usually report significant physical resistance when advancing an electrode into the cochlea, but intracochlear trauma is known to occur frequently. For example, electrodes translocate from the scala tympani to the scala vestibuli in approximately one third of cochlear implantation (CI) surgeries [Aschendorff et al., 2007; Skinner et al., 2007; Finley et al., 2008]. In previous work, we measured forces while advancing a probe representing an electrode from the scala tympani to the scala vestibuli of cadavers [Schuster et al., 2015], passing through the basilar membrane and other structures separating the vestibuli. We were not able to characterize the perceptibility of the forces recorded in these experiments because, to the best of our knowledge, no previous study has investigated surgeons' abilities to perceive electrode insertion forces during CI surgery.

Perceptual abilities are challenging to measure because they relate to subjective states of awareness which cannot be directly observed. Rigorous methods that address this measurement problem are found in the field of psychophysics [Kingdom and Prins, 2010; Jones and Tan, 2013], a discipline concerned with the relation between physical stimuli and human perception. Systematic measurement of force perception began with the work of Fechner and Weber in the 19th century [Gescheider, 2013], and psychophysical methods are now used widely in applications requiring quantification of sensory abilities. Testing pro-

\section{KARGER}

E-Mail karger@karger.com www.karger.com/aud (c) 2016 S. Karger AG, Basel

$1420-3030 / 16 / 0214-0244 \$ 39.50 / 0$
Robert F. Labadie

Vanderbilt University Medical Center 10269 Medical Center East, South Tower Nashville, TN 37232-8605 (USA)

E-Mail robert.labadie@vanderbilt.edu 
cedures used routinely in audiometry and optometry, for example, are informed by the extensive psychophysics literature.

In modern psychophysics, perception is viewed as a random process in which the perceptibility of a fixed stimulus intensity varies with repeated application of the stimulus. Perception is understood to occur in the presence of noise, including both external noise and the intrinsic noise of the sensory system. Thus, multiple measurements are needed to estimate the minimum stimulus intensity that an individual can perceive (i.e. the absolute threshold, hereafter abbreviated as the 'threshold'). Staircase procedures are one widely used method for estimating thresholds from multiple trials [Leek, 2001; Kingdom and Prins, 2010]. Typically, staircase trials begin with presentation of a large, easily perceivable stimulus intensity, which is reduced by fixed increments until the subject can no longer perceive the stimulus. The stimulus intensity is then increased by fixed steps until it is again perceptible, at which time the direction of the steps is again reversed. A threshold (quantified as a targeted performance level on a psychometric function) [Leek, 2001] is estimated by averaging all of the reversal points at which the stimulus changes direction. Often, an 'n down, 1 up' rule is used in which n successive correct responses are required before the stimulus can be decreased. The convergence of a given staircase sequence to a threshold depends on the stimulus intensities, the step sizes, the number of trials, and other factors [Garcia-Perez, 1998].

Psychophysical experiments are prone to errors caused by subjects' desire to perform well. For example, subjects may affirm the presence of a stimulus even when it is absent or imperceptibly small. Such bias is of particular concern in the present study because the subjects, who are surgeons, may interpret success at the experimental task as an indicator of occupational skill. A standard method to eliminate such bias in psychophysics experiments is the 2-alternative forced-choice (2AFC) method. Rather than replying 'yes' or 'no' to the presence of a stimulus, subjects are forced to choose between randomly assigned noise and stimulus alternatives. The effects of subjective preference are mitigated because the correct alternative is presented in a random sequence.

Much of the psychophysics literature relates to the hand and fingertips [Jones, 1986; Jones and Lederman, 2006], but few studies have examined absolute force perception thresholds under conditions comparable to CI surgery. Force thresholds have been measured in the context of diabetic neuropathy testing [Bell-Krotoski and Tomancik, 1987; Voerman et al., 1999], but the point forces applied in neuropathy testing do not resemble the distribution of pressure across a surgeon's gloved palm and fingertips when grasping a forceps to implant an electrode. Loads distributed across one or more fingertips have been tested by developers of haptic devices. Dosher and Hannaford [2005] used a forced-choice, adaptive testing protocol to measure forces applied to the fingertip with a haptic device and reported average detection thresholds ranging from 30.1 to $50.4 \mathrm{mN}$. Using a similar psychophysical testing protocol, King et al. [2010] applied forces to multiple combinations of fingertips and reported a threshold range of $27.8-34.0 \mathrm{mN}$. Baud-Bovy and Gatti [2010] transmitted force via a robot end effector to a spherical handle grasped by a subject and reported a minimum threshold of $49 \mathrm{mN}$ when the subject was allowed to move the handle to seek the direction of the force. These studies advance our understanding of the hand's sensitivity, but the configurations of these devices did not resemble the grasp and motion used in CI surgery.

In this study, we use a $2 \mathrm{AFC}$ staircase procedure to estimate the absolute force perception thresholds of a group of otologic surgeons, using an apparatus specifically designed to replicate the arm posture and forceps grasp used in CI surgery.

\section{Methods}

We recruited 12 subjects for this study by e-mail, phone, and personal communications, and stipulated that all were otolaryngologists who actively performed CI surgery in their clinical practice, or otolaryngology residents at a residency training program approved by the Accreditation Council for Graduate Medical Education (ACGME). Postgraduate year 1 residents were excluded, as they have little or no training or experience in otologic surgery in the first year of residency. The demographics of the subjects are presented in table 1. Seven subjects were residents or fellows, and 5 were attending surgeons. Two subjects were excluded from this study for reasons discussed in Results. All subjects were male. This study was approved by the Vanderbilt University Institutional Review Board, and all subjects signed informed consent after reviewing the risks and benefits of participation.

Threshold testing requires application of stimuli that have known intensities. We used Semmes-Weinstein monofilaments (SWM), which are handles embedded with a nylon monofilament (a single strand of filament), to apply forces. Each filament is calibrated to buckle when a force applied along the filament axis exceeds a particular intensity. SWM are a standard clinical tool for the assessment of cutaneous sensory function, and they are used regularly to evaluate diabetic peripheral neuropathy. They are recommended by the American Diabetes Association [Mayfield et al., 2004], and their mechanical reliability has been thoroughly demonstrated [Haloua et al., 2011]. 




Fig. 1. a An opaque curtain is placed between subject and the testing device to prevent visual observation of the experimental apparatus and the experimenter during trials. The curtain ensures that the subject experiences only tactile feedback and cannot see his or her tested hand, which may contain an experimental SWM handle (with filament) or a control handle (with the filament removed). b Top view of a seated subject. The subject's dominant hand is inserted through a slit in the curtain, which is covered with an additional flap of curtain fabric to prevent observation of the testing apparatus through gaps around the wrist. The experimenter (not shown) sits opposite from the subject. The curtain prevents the subject from visually observing the experimenter.
Table 1. Demographics of the subjects included in the analysis of force perception thresholds

\begin{tabular}{lll}
\hline & $\begin{array}{l}\text { Residents/fel- } \\
\text { lows } \\
(\mathrm{n}=6)\end{array}$ & $\begin{array}{l}\text { Attendings } \\
(\mathrm{n}=4)\end{array}$ \\
\hline $\begin{array}{l}\text { Average age, years } \\
\begin{array}{l}\text { Fellowship training completed } \\
\quad \text { Otology/neurotology }\end{array}\end{array}$ & $30.5(27-36)$ & $53.5(37-79)$ \\
$\quad \mathrm{n} / \mathrm{a}$ & $3(75)$ \\
CI surgeriatrics & $\mathrm{n} / \mathrm{a}$ & $1(25)$ \\
& $\mathrm{n} / \mathrm{a}$ & $4(3-9)$ \\
\hline
\end{tabular}

Values are presented as medians (range) or numbers (\%). All subjects were male and right-handed. $\mathrm{n} / \mathrm{a}=$ Not applicable.

Table 2. SWM were selected from the standard clinical set to span the expected range for the force threshold during CI surgery

\begin{tabular}{lc}
\hline Force, $\mathrm{g}$ & Force, $\mathrm{mN}$ \\
\hline 0.4 & 3.9 \\
0.6 & 5.9 \\
1 & 9.8 \\
1.4 & 13.7 \\
2 & 19.6 \\
4 & 39.2 \\
6 & 58.8 \\
8 & 78.5 \\
10 & 98.1 \\
15 & 147.1 \\
\hline
\end{tabular}

The SWM are presented in both grams, which are customary for SWM, and millinewton force units.
In clinical usage, a physician grasps an SWM handle and presses the filament tip into the patient's skin until the filament buckles. In our experiment, the subjects grasped SWM handles as they would hold a forceps to implant a cochlear implant electrode, with the filament in the approximate position of an electrode. By buckling the filament, a calibrated force was applied to the subjects' hands in a way that resembled the transmission of force through CI forceps (we describe how the filaments were buckled shortly).

SWM are provided in a standard set of buckling forces, which follow a logarithmic scale [Mueller, 1996]. A logarithmic scale is used because the perceived intensity of sensory stimuli is approximately proportional to the logarithm of the intensities of those stimuli. We selected a range of 8 SWM force intensities, listed in table 2, to span the anticipated threshold range of all subjects.

Prior to testing, the subjects were seated behind an opaque curtain, shown in figure 1, which prevented them from seeing both their tested hand and the experimenter. The subjects donned a suitably sized surgical glove on the dominant hand and passed this hand through a slit in the curtain, which was covered by an additional flap of fabric to prevent observation of the tested hand through the slit.

A testing device shown in figure 2, was located behind the curtain. We designed this device to cause reliable buckling of the SWM filaments and to reduce variability in the motion of the SWM handles among the subjects. Because the subjects could not see their tested hands, the testing device was needed to guide the motion of the handle between two fixed positions. Before commencing each trial, the experimenter guided the end of the SWM handle into the starting fixture of the testing device, as shown in figure $3 \mathrm{~b}$, which cradled the handle above a target surface. The distance between the starting fixture and the target surface was fixed, but the mutual height of these components above the tabletop was adjustable. The experimenter adjusted this height, as shown in figure 3a, such that the SWM handle was approximately parallel to the tabletop when the tip of the handle was in the starting fixture. 


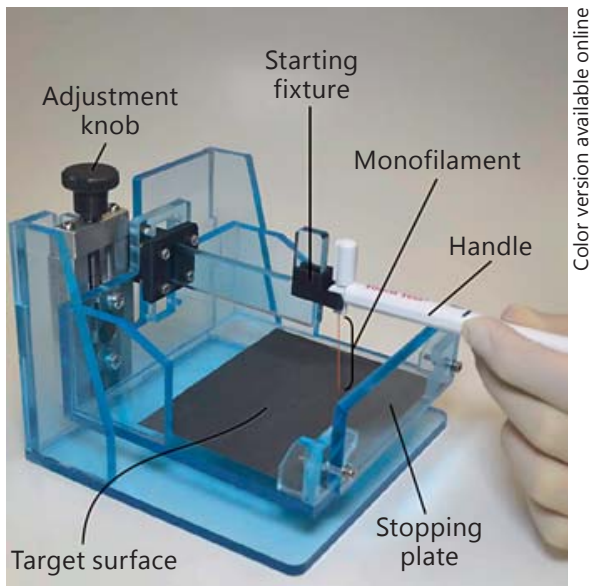

Fig. 2. Custom-built testing device used to model the advancement of forceps and transmission of contact forces during CI surgery.

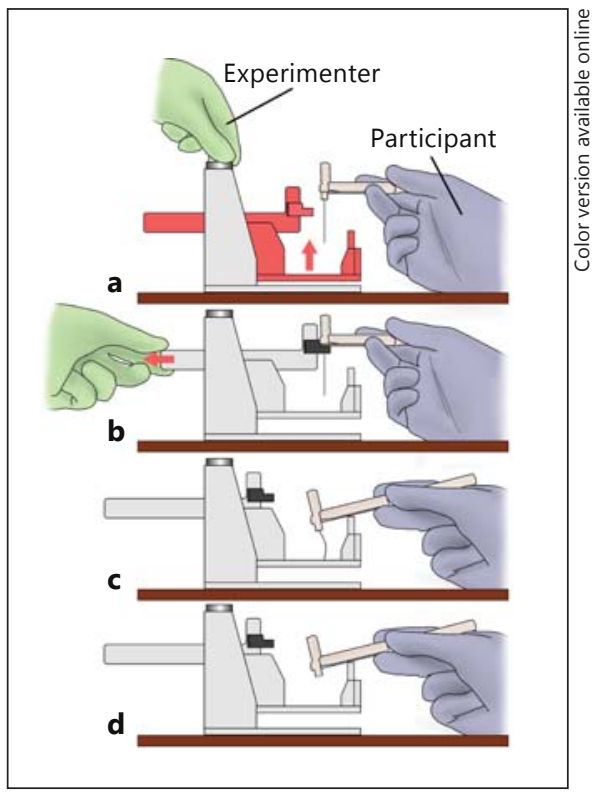

Fig. 3. a Before commencing trials, the experimenter adjusts the testing device such that the SWM handle is approximately parallel to the tabletop when it is placed in the starting fixture. $\mathbf{b}$ To begin a trial, the experimenter retracts the starting fixture and the subject lowers the SWM handle until it contacts the stopping plate. c In experimental trials the filament is buckled when the handle is in contact with the stopping plate. $\mathbf{d}$ In control trials no filament is attached to the handle.

Without a means to adjust the height of the starting fixture, the SWM handle would be initially inclined with respect to the target surface at an angle determined by the width of the subject's palm. Such initial angulation of the handle could have caused the fila-

Force Perception Thresholds in CI



Fig. 4. Plot generated from the responses of a single subject demonstrating correct and incorrect responses and the resulting staircase. Three correct responses were required before decreasing the stimulus intensity. This plot shows 3 reversal pairs consisting of 6 reversal points. A reversal point is defined as a change in direction of the plot.

ment to contact the target surface at a steep angle and possibly skid or bend rather than buckle.

To commence a trial, the experimenter pulled a handle to release the end of the SWM handle from the starting fixture, as shown in figure $3 \mathrm{~b}$. The subject then followed instructions to lower the handle until it contacted a stopping plate, a position in which the filament was buckled against the target surface, as shown in figure 3c. Alternately, if a control handle without a filament was used as discussed below, the handle rested against the stopping plate as shown in figure $3 \mathrm{~d}$. The target surface was covered in sandpaper to prevent sliding of the filament tip. The height of the stopping plate was chosen to be slightly shorter than the shortest filament length in order to stop advancement just after buckling, preventing the filament from bending severely or skidding on the target surface.

Our testing procedure with the apparatus followed a 2AFC, 3 down, 1 up (3D1U) staircase procedure. Previously, Tracey et al. [2012] evaluated the test-retest of several 2AFC procedures for SWM testing of tactile thresholds. The authors found that a testretest reliability coefficient greater than 0.80 could be achieved with as few as 2 reversal pairs using a 3D1U method. Encouraged by these results, we adapted the 2AFC-3D1U procedure to estimate surgeons' force perception thresholds.

Before commencing trials, the experimenter read instructions to each subject from a script. To implement the $2 \mathrm{AFC}$ method, a control SWM was prepared by removing the filament from a standard SWM handle, as illustrated in figure $3 \mathrm{~d}$. In each trial, the control SWM device and one SWM device from the set listed in table 2 were presented in random order. The experimenter placed an SWM handle (either a normal SWM or the control) in the subject's hand and then placed the handle in the starting fixture. After retracting the starting fixture, the experimenter read scripted instructions to the subject to begin lowering the SWM handle at the speed he would use to insert a CI electrode, until the SWM handle 
Table 3. Force perception threshold statistics computed from 10 otolaryngological surgeon subjects

\begin{tabular}{llll}
\hline & $\begin{array}{l}\text { Residents/fellows } \\
(\mathrm{n}=6)\end{array}$ & $\begin{array}{l}\text { Attendings } \\
(\mathrm{n}=4)\end{array}$ & $\begin{array}{l}\text { All } \\
(\mathrm{n}=10)\end{array}$ \\
\hline Median, $\mathrm{mN}$ & 20.4 & 26.6 & 22.3 \\
Minimum, $\mathrm{mN}$ & 10.8 & 17.4 & 10.8 \\
Maximum, mN & 33.6 & 36.5 & 36.5 \\
\hline
\end{tabular}

contacted the stopping plate. This sequence was then repeated with the second device of the 2AFC pair. Following each 2AFC pair, the subjects were instructed to report which device (the first or the second) was associated with force sensation.

Our staircase procedure began with a filament buckling strength of $39.2 \mathrm{mN}$, which we predicted would be perceivable by all subjects at all times. After 3 consecutive correct 2 AFC trials, the filament strength was decreased by 1 step in the series listed in table 2. Conversely, an incorrect response, implying an inability to perceive the presented force, was followed by an increase in filament strength in the subsequent trial. All subjects were tested with 25 pairs of filaments. A force perception threshold was calculated for each surgeon using the arithmetic mean of the log-transformed stimulus intensity values (thresholds are calculated in units in which the steps are constant) [Garcia-Perez, 1998]. One representative staircase plot is shown in figure 4 .

\section{Results}

Using the 2AFC-3D1U staircase procedure, we found a median threshold of $20.4 \mathrm{mN}$, with a range of 10.8-36.5 $\mathrm{mN}$, as listed in table 3 . All 12 subjects completed testing, but 2 ( 1 resident and 1 attending) were excluded from the analysis because their performance exceeded the range of SWM filaments given in table 2 during staircase testing. One subject exceeded the maximum strength filament in our set, and the other exceeded the weakest filament. Thus, we were not able to compute thresholds for these 2 individuals.

\section{Discussion}

Electrode insertion forces during CI surgery range from approximately 0 to $250 \mathrm{mN}$. We reported a median force perception threshold of $22.3 \mathrm{mN}$, which suggests that surgeons have the capability to perceive at least some forces in CI surgery. To date, no study has measured force perception thresholds as they pertain to CI surgery. Force thresholds for other surgical tasks have been examined [Zhou et al., 2008] but, to our knowledge, this study is the first to measure surgeons' force perception thresholds using psychophysical methods. The results may aid the design of less traumatic CI surgical techniques and devices.

Electrode insertion forces may depend on the speed of insertion, the type of electrode, the insertion tool, the intracochlear anatomy, and other factors. Furthermore, forces vary continuously during insertion, and force profiles vary from surgery to surgery. To control for the many possible sources of variation in the clinical environment, we standardized the subjects' hand motions and applied forces of constant magnitude. By applying forces abruptly and in the absence of preexisting forces, we sacrificed some fidelity to clinical conditions. Rapidly changing forces that are applied in the absence of other forces may be easier to perceive than forces that rise gradually [Jones and Lederman, 2006]. Thus, the ability to identify traumatic forces may be diminished when experienced in combination with other forces of benign origin. Before an electrode rounds the basal turn, frictional forces on the electrode are often very small, and thus our results are directly applicable to the perceptibility of forces during this phase of the surgery.

Previously, we reported a median force of $88 \mathrm{mN}$ to puncture the inner membrane of the scala tympani with a probe [Schuster et al., 2015]. Puncture of this membrane occurs when electrodes translocate from the scala tympani to the scala vestibuli, a traumatic event that occurs in approximately one third of CI surgeries. Our present results suggest that this frequent form of trauma is perceptible to surgeons, though the puncture forces we measured directly at the membrane may be higher than the forces transmitted to the hand during puncture.

\section{Conclusion}

Surgeons' force perception thresholds can be estimated using a simple, benchtop model of CI surgery. We estimated force thresholds using psychophysical testing methods, which are beneficial for handling variability and bias.

To improve the measurement of force perception thresholds, future work should aim for greater fidelity to clinical conditions. Time-varying forces could be applied by an actuator, but haptic rendering of small forces is technically challenging [see Gurari and Baud-Bovy, 2014, for an example]. Force sensors have been used to continuously measure insertion forces in both cochlear phantoms and cadaveric specimens [Majdani et al., 2010], and instrumentation could be developed to acquire in vivo 
force measurements. However, when recording forces over time, threshold estimation will be complicated by the reaction time delay between the onset of force perception and the surgeon's signal that a force has been perceived. Reaction times range from approximately 100 to $200 \mathrm{~ms}$, which would make it difficult to distinguish a perceived force from nearby values on a rapidly fluctuating force recording. Furthermore, methods will be needed to control for false positives in cadaveric or in vivo experiments. Our experimental model is easily replicable, controls for false positives, and could be used to enlarge the available data while more elaborate methods for estimating force perception thresholds in CI surgery are developed.

\section{Disclosure Statement}

This project was supported by award No. R01DC008408 from the National Institute on Deafness and Other Communication Disorders. The content is solely the responsibility of the authors and does not necessarily represent the official views of the National Institute on Deafness and Other Communication Disorders or the National Institutes of Health.

R.F. Labadie consulted for Advanced Bionics Corporation during the 2 years prior to submission of this article. The remaining authors declare that there are no conflicts of interests.

\section{References}

Aschendorff A, Kromeier J, Klenzner T, Laszig R: Quality control after insertion of the nucleus contour and contour advance electrode in adults. Ear Hear 2007;28:75S-79S.

Baud-Bovy G, Gatti E: Hand-held object force direction identification thresholds at rest and during movement; in Kappers AML, Van Erp JB, Bergmann Tiest WM, et al (eds): Haptics: Generating and Perceiving Tangible Sensations. Berlin, Springer, 2010, pp 231-236.

Bell-Krotoski J, Tomancik E: The repeatability of testing with Semmes-Weinstein monofilaments. J Hand Surg Am 1987;12:155-161.

Brito R, Monteiro TA, Leal AF, Tsuji RK, Pinna $\mathrm{MH}$, Bento RF: Surgical complications in 550 consecutive cochlear implantation. Braz J Otorhinolaryngol 2012;78:80-85.

Dosher J, Hannaford B: Human interaction with small haptic effects. Presence 2005;14:329344.

Finley CC, Holden, TA, Holden, LK, Whiting BR, Chole RA, Neely GJ, Hullar TE, Skinner MW: Role of electrode placement as a contributor to variability in cochlear implant outcomes. Otol Neurotol 2008;29:920-928.

Garcia-Perez MA: Forced-choice staircases with fixed step sizes: asymptotic and small-sample properties. Vision Res 1998;38:1861-1881.

Gescheider GA: Psychophysics: the Fundamentals, ed 3. New York, Psychology Press, 2013.

Gurari N, Baud-Bovy G: Customization, control, and characterization of a commercial haptic device for high-fidelity rendering of weak forces. J Neurosci Methods 2014;235:169_ 180.
Haloua MH, Sierevelt I, Theuvenet WJ: SemmesWeinstein monofilaments: influence of temperature, humidity, and age. J Hand Surg Am 2011;36:1191-1196.

Jones LA: Perception of force and weight: theory and research. Psychol Bull 1986;100:29.

Jones LA, Lederman SJ: Human Hand Function, ed 1. New York, Oxford University Press, 2006.

Jones LA, Tan HZ: Application of psychophysical techniques to haptic research. IEEE Trans Haptics 2013;6:268-284.

King HH, Donlin R, Hannaford B: Perceptual thresholds for single vs. multi-finger haptic interaction. IEEE Haptics Symp, Waltham, 2010, pp 95-99.

Kingdom F, Prins N: Psychophysics: a Practical Introduction, ed 1. New York, Academic Press, 2010.

Leek MR: Adaptive procedures in psychophysical research. Percept Psychophys 2001;63:1279_ 1292.

Majdani O, Schurzig D, Hussong A, Rau T, Wittkopf J, Lenarz T, Labadie RF: Force measurement of insertion of cochlear implant electrode arrays in vitro: comparison of surgeon to automated insertion tool. Acta Otolaryngol 2010;130:31-36.
Mayfield JA, Reiber GE, Sanders LJ, Janisse D, Pogach LM; American Diabetes Association: Preventative foot care in diabetes. Diabetes Care 2004;27(suppl 1):S63-S64.

Mueller MJ: Identifying patients with diabetes mellitus who are at risk for lower-extremity complications: use of Semmes-Weinstein monofilaments. Phys Ther 1996;76:68-71.

Schuster D, Kratchman LB, Labadie RF: Characterization of intracochlear rupture forces in fresh human cadaveric cochleae. Otol Neurotol 2015;36:657-661.

Skinner MW, Holden TA, Whiting BR, Voie AH, Brunsden B, Neely JG, Saxon EA, Hullar TE, Finely CC: In vivo estimates of the position of advanced bionics electrode arrays in the human cochlea. Ann Otol Rhinol Laryngol Suppl 2007;116:2-24.

Tracey EH, Greene AJ, Doty RL: Optimizing reliability and sensitivity of Semmes-Weinstein monofilaments for establishing point tactile thresholds. Physiol Behav 2012;105:982-986.

Voerman VF, van Egmond J, Crul BJ: Normal values for sensory thresholds in the cervical dermatomes: a critical note on the use of SemmesWeinstein monofilaments. Am J Phys Med Rehabil 1999;78:24-29.

Wilson BS, Dorman MF: Cochlear implants: current designs and future possibilities. J Rehabil Res Dev 2008;45:695-730.

Zhou M, Perreault J, Schwaitzberg S, Cao C: Effects of experience on force perception threshold in minimally invasive surgery. Surg Endoscop 2008;22:510-515. 